\title{
Continuous rhPTH (1-34) treatment in chronic hypoparathyroidism
}

\author{
Carmina Teresa Fuss ${ }^{1}$, Stephanie Burger-Stritt ${ }^{1}$, Silke Horn ${ }^{1}$, Ann-Cathrin Koschker ${ }^{1}$, \\ Kathrin Frey ${ }^{1}$, Almuth Meyer ${ }^{2}$ and Stefanie Hahner ${ }^{1}$ \\ 1Division of Endocrinology and Diabetology, Department of Medicine I, University Hospital Würzburg, Würzburg, \\ Germany and 2Division of Endocrinology and Diabetology, Department of Internal Medicine, Helios Klinikum Erfurt, \\ Erfurt, Germany
}

Correspondence

should be addressed

to S Hahner

Email

hahner_s@ukw.de

\section{Summary}

Standard treatment of hypoparathyroidism consists of supplementation of calcium and vitamin D analogues, which does not fully restore calcium homeostasis. In some patients, hypoparathyroidism is refractory to standard treatment with persistent low serum calcium levels and associated clinical complications. Here, we report on three patients (58-year-old male, 52-year-old female, and 48-year-old female) suffering from severe treatment-refractory postsurgical hypoparathyroidism. Two patients had persistent hypocalcemia despite oral treatment with up to $4 \mu \mathrm{g}$ calcitriol and up to $4 \mathrm{~g}$ calcium per day necessitating additional i.v. administration of calcium gluconate 2-3 times per week, whereas the third patient presented with high frequencies of hypocalcemic and treatment-associated hypercalcemic episodes. S.c. administration of rhPTH (1-34) twice daily (40 $\mu \mathrm{g} /$ day) or rhPTH (1-84) (100 $\mu \mathrm{g} /$ day) only temporarily increased serum calcium levels but did not lead to long-term stabilization. In all three cases, treatment with rhPTH (1-34) as continuous s.c. infusion via insulin pump was initiated. Normalization of serum calcium and serum phosphate levels was observed within 1 week at daily 1-34 parathyroid hormone doses of $15 \mu \mathrm{g}$ to $29.4 \mu \mathrm{g}$. Oral vitamin D and calcium treatment could be stopped or reduced and regular i.v. calcium administration was no more necessary. Ongoing efficacy of this treatment has been documented for up to 7 years so far. Therefore, we conclude that hypoparathyroidism that is refractory to both conventional treatment and s.c. parathyroid hormone (single or twice daily) may be successfully treated with continuous parathyroid hormone administration via insulin pump.

\section{Learning points:}

- Standard treatment of hypoparathyroidism still consists of administration of calcium and active vitamin D.

- Very few patients with hypoparathyroidism also do not respond sufficiently to standard treatment or administration of s.c. parathyroid hormone once or twice daily.

- In those cases, continuous s.c. administration of parathyroid hormone via insulin pump may represent a successful treatment alternative.

\section{Background}

Hypoparathyroidism is characterized by hypocalcemia and hyperphosphatemia caused by the absence or deficiency of PTH. The most common cause of hypoparathyroidism is postsurgical after thyroidectomy (1). Patients typically suffer from paraesthesia, muscle cramps and tetany. Longterm complications consist of nephrocalcinosis and renal insufficiency, as well as neurological manifestations, for example, seizures and basal ganglia calcifications (2). Hypoparathyroidism is an endocrine disease whose treatment is currently not based on replacement of the missing hormone. Instead, standard therapy consists of calcium and vitamin D supplementation which 
generally restores serum calcium levels to normal ranges. However, in some cases, patients suffer from severe hypoparathyroidism resistant to conventional treatment regimens.

Parathyroid hormone preparations (rhPTH (1-84) or rhPTH (1-34)) administered as single s.c. injections were introduced in the market for treatment of osteoporosis several years ago. Since 2017, rhPTH 1-84 is also available for patients with treatment-refractory hypoparathyroidism in Germany. Recent trials studied substitution therapy in patients with primary hypoparathyroidism. Under treatment with PTH, serum calcium levels could be stabilized, sometimes leading to hypercalcemic episodes if PTH application followed a permanent treatment regimen, whereas skipping of PTH injection causes fast reduction of serum calcium. Patients also profit from the reduction of elevated serum phosphate levels (3). In a recent study, PTH administration was safe, tolerable and effective over a period of 5 years (4).

Because PTH injection once a day often does not lead to stable calcium concentration, PTH was applied multiple times a day leading to less fluctuations of serum calcium. Especially, PTH delivery via pump systems seems to provide good calcium control, normalization of boneremodeling and reduction of daily PTH doses over the course of 3 months (5).

Here we report on three patients with uncontrolled postsurgical hypoparathyroidism. Despite high-dose oral calcium and calcitriol supplementation, frequent i.v. calcium administration, as well as daily rhPTH (1-34) or rhPTH (1-84) treatment, severe hypocalcemic episodes persisted. In all three cases, hypoparathyroidism could only be controlled by continuous s.c. administration of rhPTH (1-34). All patients gave written informed consent.

\section{Case presentation}

\section{Patient 1}

A 52-year-old female patient was referred to our Endocrinology Service for further evaluation and treatment of severe hypocalcemia. She suffered from postsurgical hypoparathyroidism after thyroidectomy and parathyroidectomy due to multinodular goiter in 1999. The patient presented with paraesthesia of upper and lower limbs, hand tremor and recurrent epileptic seizures under treatment with daily doses of 1.5 to $3 \mathrm{~g}$ calcium, $1.5 \mathrm{~g}$ magnesium and $4 \mu \mathrm{g}$ calcitriol. In addition, she received i.v. calcium administrations two to three times per week via a central-venous port-system and 100000 IU cholecalciferol intramuscularly twice a week. Serum calcium levels of 1.5-1.7 $\mathrm{mmol} / \mathrm{L}$ (reference range (RR): $2.2-2.7 \mathrm{mmol} / \mathrm{L})$, hyperphosphatemia (1.77 $\mathrm{mmol} / \mathrm{L}, \mathrm{RR}: 0.8-1.3 \mathrm{mmol} / \mathrm{L})$, hypocalciuria (1.45-3.72 $\mathrm{mmol} / 24 \mathrm{~h}$, reference value: $4.00-8.00 \mathrm{mmol} / 24 \mathrm{~h}$ ) and low 1,25-OH-vitamin-D levels were found. To rule out a potential malabsorption of calcitriol, D-xylose absorption test and endoscopic procedures were performed, showing no abnormalities. We started the additional off-label treatment with $100 \mu \mathrm{g}$ rhPTH (1-84) daily and s.c. injection of $1 \mu \mathrm{g}$ calcitriol/day which together with up to $3 \mathrm{~g}$ oral calcium supplementation resulted in short-term stabilization of serum calcium levels $(2.2 \mathrm{mmol} / \mathrm{L})$. Four months later she was readmitted because of severe recurrent hypocalcemia $(1.6 \mathrm{mmol} / \mathrm{L})$ requiring i.v. calcium supplementation. Due to low 1,25-OH-vitamin-D levels (15 ng/L, RR: $35-80 \mathrm{ng} / \mathrm{L})$ and normal 25-OH-vitamin-D levels $(55.8 \mu \mathrm{g} / \mathrm{L}, \mathrm{RR}: 30-70$ $\mu \mathrm{g} / \mathrm{L})$, the daily s.c. calcitriol dosage was increased to 2 $\mu \mathrm{g}$. Additional treatment with PTH (1-84) $100 \mu \mathrm{g} /$ day was switched to $20 \mu \mathrm{g}$ PTH (1-34) daily with division into up to five single injections distributed over the day. However, these adjustments still did not provide amelioration of serum calcium, phosphate and vitamin D levels. Our patient still experienced severe paraesthesia and epileptic seizures and developed slight renal insufficiency (GFR $\left.(\mathrm{MDRD})=64 \mathrm{~mL} / \mathrm{min} / 1.73 \mathrm{~m}^{2}\right)$. Renal ultrasound showed no signs of nephrocalcinosis. Potential compliance issues were ruled out by supervised drug intake. All treatment modifications described previously were conducted during several inpatient visits of up to 8 weeks during which the patient's therapy was directly observed, PTH was administered by trained healthcare professionals to rule out poor adherence or errors in administration and serum calcium was measured every 1 to 3 days.

\section{Patient 2}

A 58-year-old male had developed hypoparathyroidism after thyroidectomy due to multinodular goiter in 2008 . He presented with paraesthesias of his face, arms and legs as well as muscle cramps of the hands. He further suffered from intermittent nausea and vomiting. He also had to be treated at the intensive care unit twice because of loss of consciousness and tetanies caused by hypocalcemic crises. On admission, the patient was under treatment with $1.5 \mu \mathrm{g}$ calcitriol/day, $4 \mathrm{~g}$ calcium/ day and magnesium. The patient did not experience any gastrointestinal symptoms under high calcium doses. Low serum calcium of $1.2 \mathrm{mmol} / \mathrm{L}$ and increased serum 
phosphate of $1.87 \mathrm{mmol} / \mathrm{L}$ were found. By addition of $20 \mu \mathrm{g} \mathrm{rhPTH} \mathrm{(1-34),} \mathrm{increasing} \mathrm{calcitriol} \mathrm{dosage} \mathrm{to} 4 \mu \mathrm{g} /$ day and adding $3000 \mathrm{IU}$ vitamin D/day, calcium and phosphate levels improved, but did not fully normalize. Therefore, rhPTH (1-34) dosage was increased to two daily doses administered in the morning and evening and calcium i.v. was administered weekly. Despite this intensified therapy regimen, the highest measured serum calcium level was found to be still low $(1.9 \mathrm{mmol} / \mathrm{L})$ and clinical symptoms recurred only few weeks after changes in therapy had been made.

\section{Patient 3}

The third patient (48 years, female) also developed postsurgical hypoparathyroidism after thyroidectomy due to symptomatic goiter in 1983. In 1999, she was admitted to the hospital because of severe treatmentassociated hypercalcemia requiring dialysis. Since 2002, however, frequent admissions for hypocalcemic episodes had been necessary. Hypocalcemia was assumed to have been caused by recurrent diarrhea due to fructose intolerance. Because of poor calcium control, she received rhPTH (1-34) treatment starting in 2004. However, even during treatment with $40 \mu \mathrm{g}$ rhPTH (1-34) daily $(2 \times 20 \mu \mathrm{g})$ and $3 \mu \mathrm{g}$ calcitriol/day, calcium levels highly fluctuated. To improve the patient's quality of life, she was referred to us for initiation of continuous s.c. rhPTH (1-34) administration.

\section{Treatment and outcome}

S.c. rhPTH (1-34) (Forsteo, $20 \mu \mathrm{g} / 80 \mu \mathrm{L}$, Lilly) was diluted with Aqua destillata (Braun, Melsungen, Germany) and administered continuously via Omnipod pump (patient 1, Insulet Corporation, Acton, Massachusetts, USA), Medtronic pump (patient 2, Medtronic GmbH, Meerbusch, Germany) and Vibe pump (patient 3, Animas, Johnson\&Johnson Medical $\mathrm{GmbH}$ ). In patient 1 , initial infusion rate was set at $10.8 \mu \mathrm{g}$ PTH 1-34/24 $\mathrm{h}$ and further adapted to $15.3 \mu \mathrm{g} / 24 \mathrm{~h}$, whereas patient 2 immediately received a starting dose of $15 \mu \mathrm{g} / 24 \mathrm{~h}$. In patient 3, rhPTH (1-34) treatment was started at a dosage of $25.2 \mu \mathrm{g} / \mathrm{day}$. After intermittent dose reduction, she showed stable calcium levels at a slightly increased dose of $29.4 \mu \mathrm{g} /$ day. Within 1 week after start of pump therapy, normalization of serum calcium and phosphate levels could be observed in all three patients (Figs 1, 2 and 3). Clinical symptoms gradually improved. Frequency of seizures decreased (patient 1) and no more hypoand hypercalcemic crises occurred (patient $2+$ patient $3)$. Under continuous administration of rhPTH (1-34) additional medication could be reduced to $1000 \mathrm{IU}$ cholecalciferol/day (patient 1), $20000 \mathrm{IU}$ cholecalfciferol weekly + $80 \mathrm{mg}$ magnesium/day (patient 2) and calcitriol $0.5 \mu \mathrm{g} /$ day+calcium $500 \mathrm{mg} /$ day (patient 3). For the first time, in all three patients, we were able to control a severe therapy refractory hypoparathyroidism with very low rhPTH (1-34) doses for 7 (patient 1), 6.5

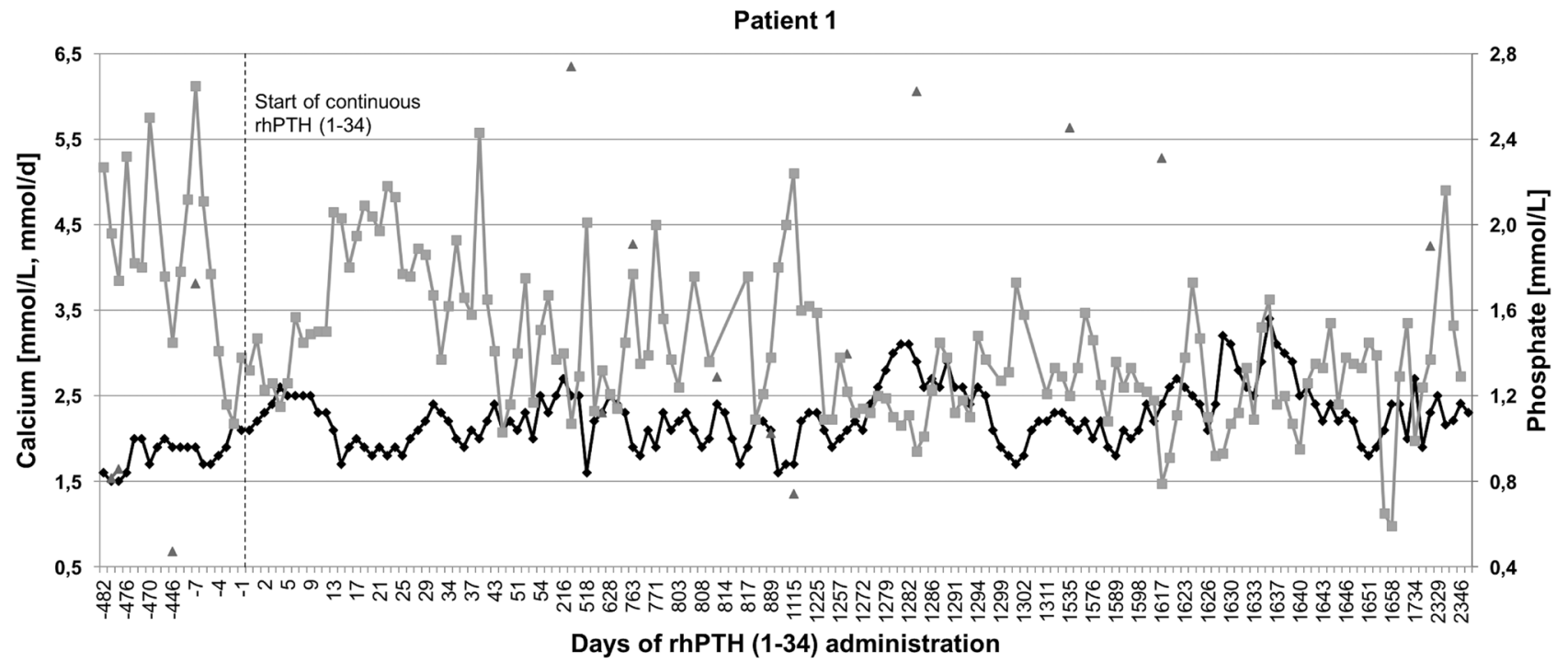

$\rightarrow$ Calcium $[\mathrm{mmol} / \mathrm{L}] \quad \leadsto$ Urinary Calcium $\quad \rightarrow$-Phosphate $[\mathrm{mmol} / \mathrm{L}]$

Figure 1

Serum calcium, phosphate and urinary calcium levels before and during rhPTH (1-34) - pump therapy in patient 1. 


\section{Patient 2}

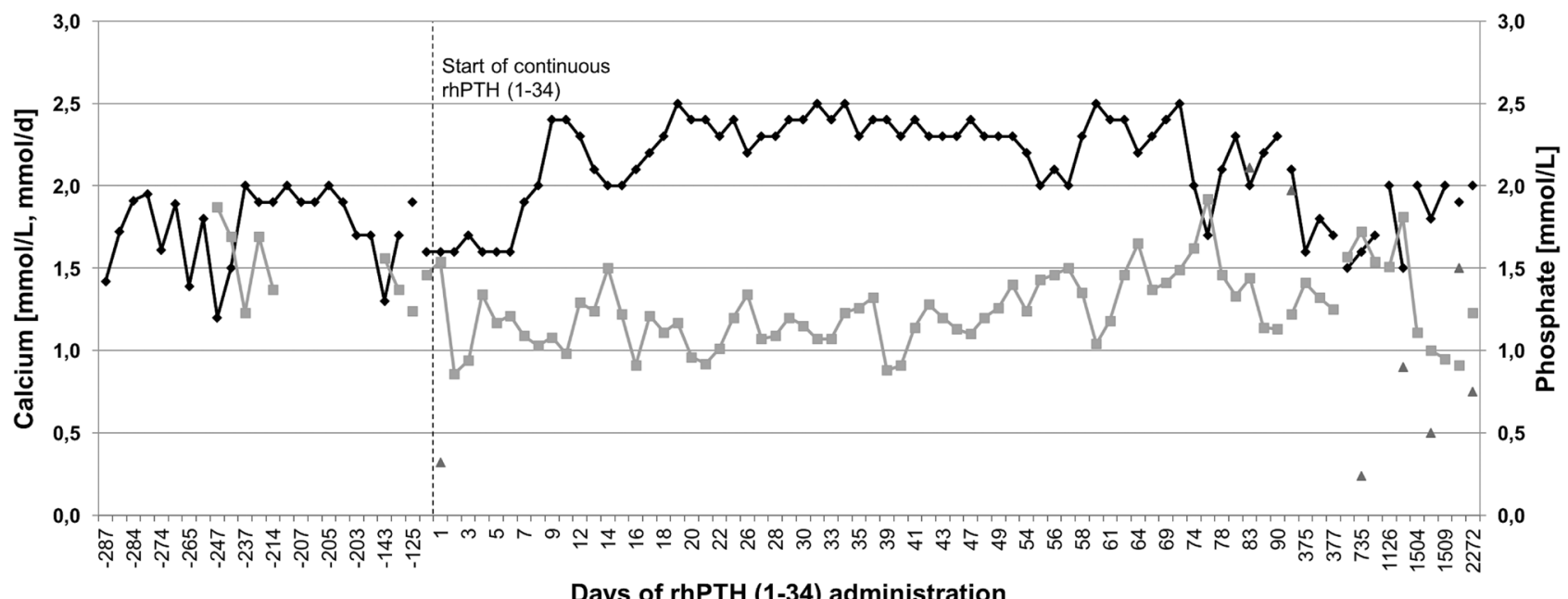

Days of rhPTH (1-34) administration

$\rightarrow$ Calcium $[\mathrm{mmol} / \mathrm{L}] \quad-$-Phosphate $[\mathrm{mmol} / \mathrm{L}] \quad \Delta$ Urinary Calcium $[\mathrm{mmol} / \mathrm{d}]$

Figure 2

Serum calcium, phosphate and urinary calcium levels before and during rhPTH (1-34) - pump therapy in patient 2.

(patient 2) and 5.5 (patient 3) years, respectively, without observing significant side effects. After treatment with continuous PTH administration for 4.5 years, patient 1 presented with severe hypercalcemia (serum calcium $3.4 \mathrm{mmol} / \mathrm{L}$ ) requiring dialysis. Hypercalcemia persisted after discontinuation of rhPTH (1-34) and oral calcium and vitamin D. The patient's history revealed the presence of sarcoidosis. Therefore, treatment with oral prednisone was initiated resulting in rapid decline in serum calcium levels due to reduced sarcoidosis activity. After reinitiation of continuous rhPTH (1-34) administration, calcium and phosphate levels could be maintained stable.

Twenty-four-hour urinary calcium was measured in patient 1 and 3 before and in all patients during treatment with continuous rhPTH (1-34). None of the patients showed hypercalciuria during administration of rhPTH (1-34), but rather a tendency toward a reduction of urinary calcium levels, whereas periodic increases in

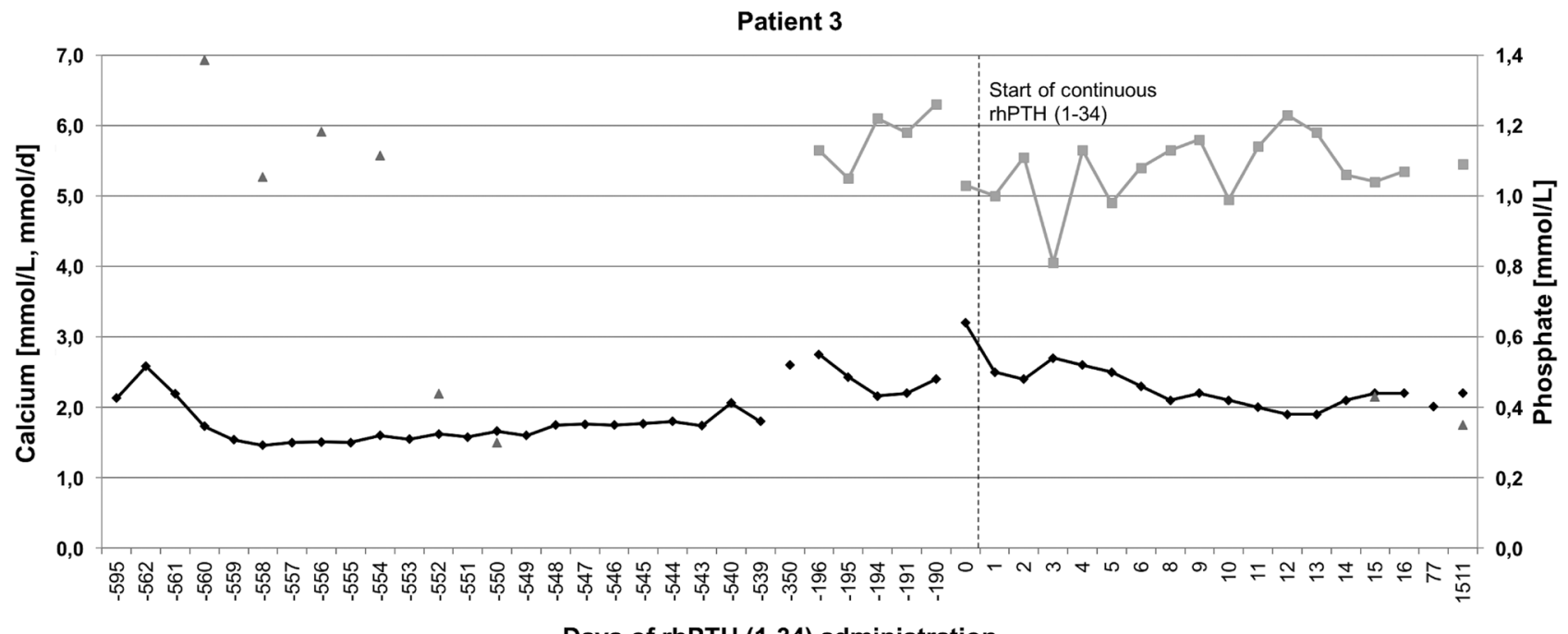

Days of rhPTH (1-34) administration

$\rightarrow$ Calcium $[\mathrm{mmol} / \mathrm{L}] \quad \rightarrow$ Urinary Calcium $[\mathrm{mmol} / \mathrm{d}] \quad-\quad \rightarrow-$ Phosphate $[\mathrm{mmol} / \mathrm{L}]$

Figure 3

Serum calcium, phosphate and urinary calcium levels before and during rhPTH (1-34) - pump therapy in patient 3. 
calcium excretion in patient 1 can most likely be attributed to periods of hypercalcemia due to active sarcoidosis.

In the long-term period, single episodes with hypocalcemia during stressful events or infectious episodes were noted in all three cases, requiring intermittent dose increase of $\mathrm{rhPTH}(1-34)$ and additional oral calcium intake.

Regarding treatment safety, in all patients, creatinine levels and glomerular filtration rate remained stable. Renal ultrasound was performed every 1 to 2 years. Nephrocalcinosis was present in patient 3 prior to initiation of continuous PTH therapy, whereas patient 1 and 2 did not develop nephrocalcinosis during PTH treatment. Additionally, bone density measurement and skeletal scintigraphy were carried out every 1 to 2 years in patient 1 and 2 revealing neither significant changes in bone mass nor skeletal lesions over time.

\section{Discussion}

We describe three cases in which continuous s.c. administration of rhPTH (1-34) via insulin pump was the only effective way to achieve long-term stabilization of calcium homeostasis and clinical symptoms of postsurgical hypoparathyroidism.

In the majority of patients with hypoparathyroidism, hypocalcemia and associated symptoms can be treated sufficiently by active vitamin D and calcium supplementation. However, there are some limitations to this current standard treatment, especially regarding the frequent risk of hypercalcemia and hyperphosphatemia, hypercalciuria, potentially leading to nephrocalcinosis and renal dysfunction, as well as persistent mood impairment (1).

More recently, treatment with rhPTH (1-84) or rhPTH (1-34) as replacement therapy has been evaluated. Previous studies show stabilization of serum calcium and phosphate levels, reduction of calcium and vitamin D supplementation $(3,4)$. In our cases, single s.c. injections were not sufficient to restore calcium and phosphate homeostasis over a longer time period. However, we were able to normalize serum calcium and phosphate levels by continuous s.c. rhPTH (1-34) administration, reducing severe clinical manifestations in all three patients without causing hypercalcemic episodes and hypercalciuria.

This phenomenon has also been observed in two other patients, both suffering from chronic vitamin D unresponsive hypoparathyroidism which could only be sufficiently treated with continuous PTH administration, but not with PTH injections $(6,7)$. PTH therapy via insulin pump vs injections has previously been evaluated. Winer et al. compared twice-daily s.c. injections to pump treatment, both administered for 3 months followed by crossover, in 12 children and young adults with congenital hypoparathyroidism as well as in 8 adults with chronic hypoparathyroidism. In both groups, rhPTH (1-34) pump led to less fluctuation in serum calcium, greater reduction of urine calcium, significant reduction of PTH dose and magnesium supplements as well as normalization of bone turnover markers $(5,8)$. Therefore, pump treatment currently provides the closest approach to physiological calcium homeostasis.

Regarding safety and risk evaluation, development of osteosarcoma after long-term treatment with PTH in rats has been noted (9). Until now, based on data regarding PTH treatment in osteoporosis, there is no evidence of increased risk for osteosarcoma in humans (10). In our patients, no adverse events have been observed over a course of up to 7 years.

In conclusion, in cases where even PTH injections do not allow sufficient disease control, long-term PTH pump therapy represents an alternative option, providing longterm stabilization of calcium and phosphate homeostasis.

\section{Declaration of interest}

C T F is a member of the Advisory board and speaker engagement - Shire/ Takeda. The other authors have nothing to disclose.

\section{Funding}

This study was supported by the Interdisciplinary Center for Clinical Research (IZKF) at the University of Würzburg (Z-2/77).

\section{Author contribution statement}

C T F, A M and S Horn performed data collection. C T F and S Hahner drafted the manuscript. C T F, S B S, A M, A C K, S Horn and S Hahner revised the manuscript content. C T F, S B S, A M, A C K, S Horn and S Hahner approved the final version of the manuscript. C T F, S B S, A M, A C K, S Horn and S Hahner were involved in the care of the patients.

\section{References}

1 Bilezikian JP, Khan A, Potts Jr JT, Brandi ML, Clarke BL, Shoback D, Jüppner H, D'Amour P, Fox J, Rejnmark L, et al. Hypoparathyroidism in the adult: epidemiology, diagnosis, pathophysiology, targetorgan involvement, treatment, and challenges for future research. Journal of Bone and Mineral Research 201126 2317-2337. (https://doi. org/10.1002/jbmr.483)

2 Shoback DM, Bilezikian JP, Costa AG, Dempster D, Dralle H, Khan AA, Peacock M, Raffaelli M, Silva BC, Thakker RV, et al. Presentation of hypoparathyroidism: etiologies and clinical features. Journal of Clinical Endocrinology and Metabolism $20161012300-2312$. (https://doi.org/10.1210/jc.2015-3909) 
3 Mannstadt M, Clarke BL, Vokes T, Brandi ML, Ranganath L, Fraser WD, Lakatos P, Bajnok L, Garceau R, Mosekilde L, et al. Efficacy and safety of recombinant human parathyroid hormone (1-84) in hypoparathyroidism (REPLACE): a double-blind, placebo-controlled, randomised, phase 3 study. Lancet: Diabetes and Endocrinology 20131 275-283. (https://doi.org/10.1016/S22138587(13)70106-2)

4 Mannstadt M, Clarke BL, Bilezikian JP, Bone H, Denham D, Levine MA, Peacock M, Rothman J, Shoback DM, Warren ML, et al. Safety and efficacy of 5 years of treatment with recombinant human parathyroid hormone in adults with hypoparathyroidism. Journal of Clinical Endocrinology and Metabolism 2019104 5136-5147. (https:// doi.org/10.1210/jc.2019-01010)

5 Winer KK, Zhang B, Shrader JA, Peterson D, Smith M, Albert PS \& Cutler GB. Synthetic human parathyroid hormone 1-34 replacement therapy: a randomized crossover trial comparing pump versus injections in the treatment of chronic hypoparathyroidism. Journal of Clinical Endocrinology and Metabolism 201297 391-399. (https://doi. org/10.1210/jc.2011-1908)

6 Diaz-Soto G, Mora-Porta M, Nicolau J, Perea V, Halperin I \& Puig-Domingo M. Efficacy and safety of long term treatment of unresponsive hypoparathyroidism using multipulse subcutaneous infusion of teriparatide. Hormone and Metabolic Research 201244 708-710. (https://doi.org/10.1055/s-0032-1308971)

7 Puig-Domingo M, Diaz G, Nicolau J, Fernandez C, Rueda S \& Halperin I. Successful treatment of vitamin D unresponsive hypoparathyroidism with multipulse subcutaneous infusion of teriparatide. European Journal of Endocrinology 2008159 653-657. (https://doi.org/10.1530/EJE-08-0269)

8 Winer KK, Fulton KA, Albert PS \& Cutler Jr GB. Effects of pump versus twice-daily injection delivery of synthetic parathyroid hormone 1-34 in children with severe congenital hypoparathyroidism. Journal of Pediatrics 2014165 556.e1-563.e1. (https://doi.org/10.1016/j.jpeds.2014.04.060)

9 Jolette J, Wilker CE, Smith SY, Doyle N, Hardisty JF, Metcalfe AJ, Marriott TB, Fox J \& Wells DS. Defining a noncarcinogenic dose of recombinant human parathyroid hormone 1-84 in a 2-year study in Fischer 344 rats. Toxicologic Pathology 200634 929-940. (https://doi. org/10.1080/01926230601072301)

10 Andrews EB, Gilsenan AW, Midkiff K, Sherrill B, Wu Y, Mann BH $\&$ Masica D. The US postmarketing surveillance study of adult osteosarcoma and teriparatide: study design and findings from the first 7 years. Journal of Bone and Mineral Research 201227 2429-2437. (https://doi.org/10.1002/jbmr.1768)

Received in final form 26 March 2020

Accepted 21 April 2020 